\title{
Field-in-Field Technique Improves the Dosimetric Outcome of Treatment Plans Compared with the Three-Dimensional Conformal Radiation Therapy for Esophageal Cancer Radiotherapy
}

\author{
Behnam GHADIMI ${ }^{1}$, Nasrollah JABBARI ${ }^{2}$, Laya KARIMKHANI ${ }^{3}$, Kamal MOSTAFANEZHAD ${ }^{3}$ \\ ${ }^{1}$ Urmia University of Medical Sciences, Department of Medical physics and Imaging \\ ${ }^{2}$ Urmia University of Medical Sciences, Solid Tumor Research Center, Department of Medical Physics and Imaging \\ ${ }^{3}$ Omid Research and Treatment Center, Urmia, IRAN
}

\begin{abstract}
The aim of this study was to evaluate and compare the dosimetric parameters of three-dimensional conformal radiotherapy (3D-CRT) and field-in-field (FIF) techniques for patients with esophageal cancer. Twenty (20) patients with esophageal cancer participated in this study. Two planning techniques (FIF and 3D-CRT) were generated for each patient by the TiGRT treatment planning system. Two indices namely: Dose Homogeneity Index $(\mathrm{DH})$ and Conformity index $(\mathrm{Cl})$, as well as maximum dose, mean dose, minimum dose, dose received by $2 \%$ of the target volume $\left(D_{2}\right)$; dose received by $98 \%$ of the target volume $\left(D_{98}\right)$; volume received greater than $107 \%$ of the prescribed dose ( $V>107 \%)$; volume received less than 95\% of the prescribed dose $(\mathrm{V}<95 \%)$; Dose received by organ at risks (OARs) and total Monitor Unit (MUs) were used for the comparison. The mean values were then compared using paired sample t-test. The FIF technique reduced the maximum dose and mean dose in the planning target volume (PTV). The FIF technique had higher D98, lower $\mathrm{D}_{2}$ and $\mathrm{V}<95 \%$. The FIF plan recorded a better DHI than the 3D-CRT technique. However, the FIF did not show any significant differences in minimum dose, $\mathrm{V}>107 \%, \mathrm{Cl}$ and MUs compared with the 3D-CRT technique. In addition, the FIF technique demonstrated reduced dose received by the OARs in the treated region. The FIF technique enables better dose distribution in the PTV and reduces dose to OARs in esophageal radiotherapy.
\end{abstract}

Keywords: Radiotherapy, Esophageal cancer, Dosimetric parameters, Field in field technique, 3D-CRT technique

\section{INTRODUCTION}

Cancers are among the most common causes of death all over the world. It is estimated that the overall incidence of all types of cancers will increase by $45 \%$ in developed countries by $2030 .{ }^{1}$ Esophageal carcinoma (EsC) is the eighth most prevalent cancer and the sixth most prevalent cause of mortality due to cancer worldwide. ${ }^{2}$ EsC is one of the least studied and deadliest cancers worldwide because of its profound aggressive nature and poor survival rate. ${ }^{3}$

Surgery remains the main treatment modality in very early esophageal cancer. However radiotherapy plays an important role in patients with stage I-IVA esophageal cancer either as chemoradiotherapy followed by surgery or as definitive chemo- radiotherapy. 4,5 Radiation therapy alone results in poor local control and survival. Adjuvant radiation after resection has been used to amend local control and survival. ${ }^{6}$ In comparison with surgery alone, randomized trials have shown no significant overall survival (OS) benefit for postoperative radiation therapy. ${ }^{7,8}$

Three-dimensional conformal radiotherapy (3DCRT) is widely and routinely utilized for the treatment of different malignancies in the body. ${ }^{6}$ In EsC radiotherapy treatment planning, radiation fields are defined by employing anteroposterior (AP) and posteroanterior (PA) fields or by three fields in the esophageal cancer region and special filters or cerrobend blocks are placed in the path of a beam to achieve a uniform dose distribution inside the target volume. 
Wedge filter is the commonly used beam modifying contrivance that reduces the radiation intensity progressively across the beam, besides it is used to compensate for the missing tissues. One of the disadvantages of the wedge is the extra scatter which emanates from the wedge and may be integrated into the peripheral dose. In areas like the abdomen and thorax, the utilization of wedge provides only minimal improvement in the dose inhomogeneity. It reduces the effect of low energy $\mathrm{x}$-rays in the megavoltage beams and causes a beam hardening effect which can alter the depth dose at larger depths. ${ }^{9,10}$

A great progress in 3D-CRT has been achieved with the introduction of multileaf collimators (MLCs). ${ }^{9-11}$ The field-in-field (FIF) technique is a radiotherapy technique, and is also known as forward intensity-modulated radiotherapy (IMRT). The aim of the forward IMRT is to increase dose homogeneity in the target volume while decreasing the absorbed dose in the irradiated tissues outside the targeted tissue. There are other reports on the utilization of the FIF technique to improve dose distribution. ${ }^{12,13}$ Forward planning has been routinely utilized in the treatment of breast cancer and shown to give better dosimetric results than the conventional wedge fields. Due to the widespread utilization of conformal radiotherapy in developing countries, the authors decided to compare two conformal radiotherapy techniques in the field of dosimetric parameters. There is scarce literature on the utilization of the FIF technique at other sites. This study was designed to evaluate the feasibility of the FIF technique for esophageal cancers.

\section{PATIENTS AND METHODS}

Twenty (20) patients with esophageal cancer, candidates for pre-operative radiotherapy, were selected for this study. There was no sex and age limitation. The patients were immobilized with a thermoplastic mask while they were in supine position with hands lifted above their heads. Thereafter, all the patients underwent CT scanning with a 64 slice Philips Brilliance system for 3-mm slice thicknesses; then, CT datasets were transferred to a TiGRT treatment planning system ${ }^{14}$ through a DICOM network. The radiation oncologist then contoured the gross tumor volume (GTV), planning target volume (PTV) and organ at risks (OARs) on the planning CT slices, according to the guidelines of the International Commission of Radiation Units and Measurements (ICRU) $)^{15,16}$ as well as Perez \& Brady's principles and practice of radiation oncology. ${ }^{17}$ In PTV contouring, the radiation oncologist used the patients last diagnostic CT-scan and endoultrasonography (EUS). In some patients, PET imaging is also useful in defining PTV. EUS is the best modality for defining both the longitudinal and radial extent of the primary tumor. ${ }^{18}$ The radiation physicist performed the plan by the treatment planning system for a single energy linear accelerator (Siemens Primus) equipped with 51 pairs of multileaf collimators (MLC).

For all the cases, two plans were generated: a 3DCRT and a field-in-field based treatment plan. The fields of both techniques covered the entire PTV. The lymph nodes were included in the radiation target volume. Conforming field borders that allow the dosimetric coverage of PTV should be devised (usually another 5 to $10 \mathrm{~mm}$ to the field edge). The prescribed dose for the PTV was 50.40 Gy in 28 fractions with $6 \mathrm{MV} \mathrm{x}$-ray. The prescribed dose was carried in two steps. In the first step, parallel opposed beams (AP/PA) were delivered 30.60 $\mathrm{Gy} / 17 \mathrm{fr}$, then three field techniques (AP/LPO/ RPO) were used for dose delivery $(19.80 \mathrm{~Gy} / 11$ fr). This method of dose delivery causes better dose homogeneity with less normal tissue complications. Physical wedge was not used in 3D-CRT plans. In the FIF technique, several less-weighted fields with a small treatment portal size were selected to optimize dose distributions in the main fields that were used for 3D-CRT. Through a trial and error process, the optimized FIF plans were determined by evaluating the 3-D dose distribution and dose-volume histogram. Several subfields were merged into the main field, including several multileaf collimator segments for sequential irradiation. Through the use of beams eye view, high dose regions above $105 \%$ of the maximum dose, were shielded with MLCs in the steps of 5\% dose level (Figure 1). The weights of the MLC segments were adjusted manually to reduce the hotspots until an optimal dose distribution, with better dose homogeneity, was achieved inside the target volume. Both plans were evaluated and compared for the 


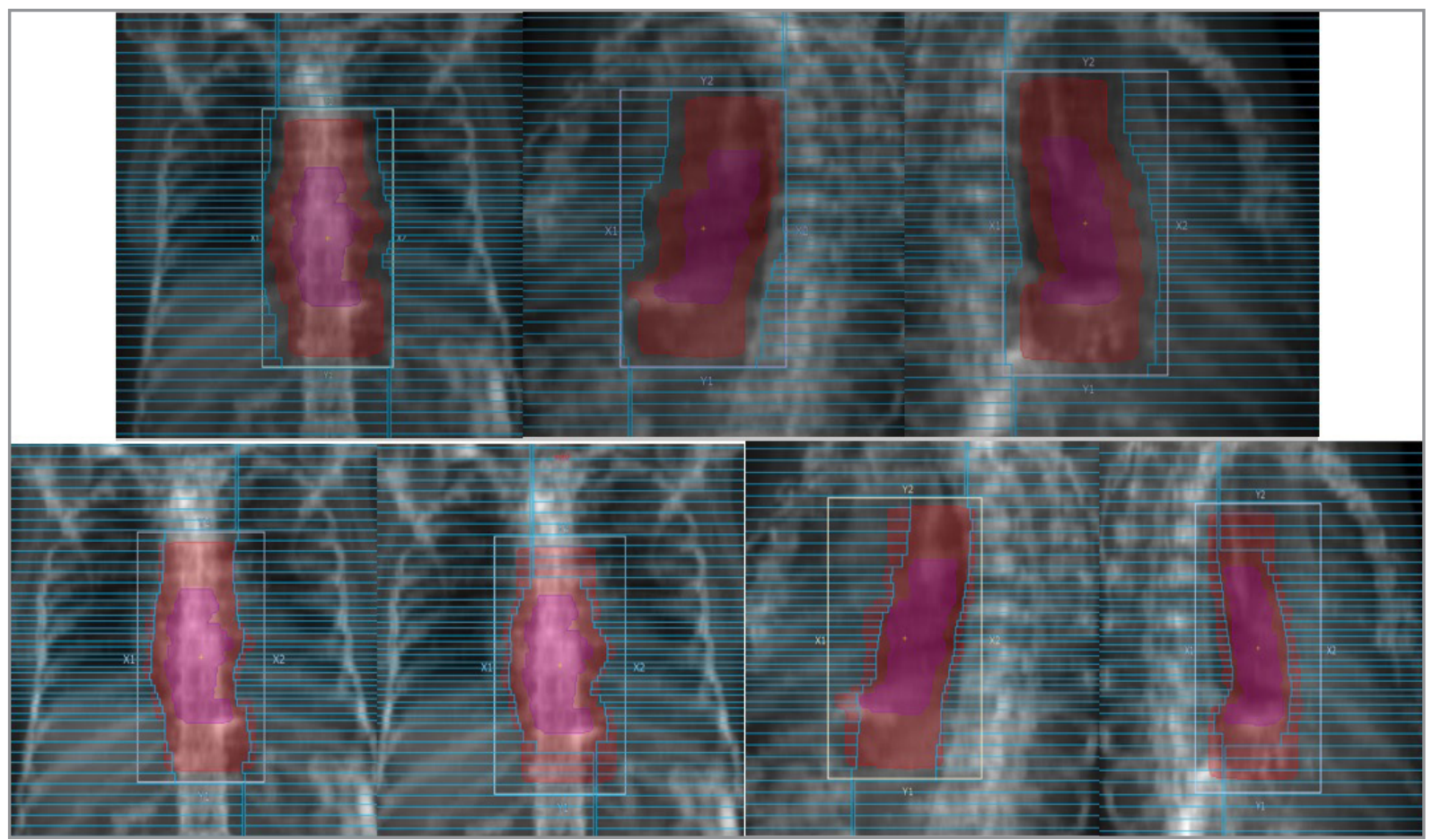

Figure 1. Main fields (top row pictures) and subfields (bottom row pictures) for removing hot points in patients with esophageal cancer.

following: mean dose, maximum dose, minimum dose; dose received by $2 \%$ of the target volume $\left(D_{2}\right)$; dose received by $98 \%$ of the target volume $\left(\mathrm{D}_{98}\right)$; volume received greater than $107 \%$ of the prescribed dose $(\mathrm{V}>107 \%)$; volume received less than $95 \%$ of the prescribed dose $(\mathrm{V}<95 \%)$; total Monitor Unit (MUs); conformity index (CI) and homogeneity index (HI). CI represents the ratio of volume enclosed by the prescription isodose to the target volume; and $\mathrm{CI}$ values ranging from $0-1$. A higher $\mathrm{CI}$ value indicates higher dose conformity to the target. ${ }^{19} \mathrm{HI}$ is defined as follows. Lower HI values indicate a more homogeneous dose distribution and doses to organ at risks. ${ }^{20}$

$$
\mathrm{HI}=\frac{\mathrm{D}_{2}-\mathrm{D}_{98}}{\mathrm{D}_{\text {prescrition }}} \times 100 \%
$$

\section{Statistical Analysis}

Statistical analysis was conducted using SPSS version 20.0. The normality of the data was assessed using the Kolmogorov-Smirnov (K-S) test. After verification of the data with normality test, the paired sample t-test was used to compare the mean values of the parameters between the two groups of patients. $p$ value $<0.05$ was considered to be statistically significant.

\section{RESULTS}

The demographic characteristics of the patients under study and PTV volumes are given in Table 1. The dose-volume histogram (DVHs) comparisons of the wedge field technique versus FIF in a typical case of esophageal cancer are presented in Figure 2. The isodose distributions of 3D-CRT and FIFbased treatment planning in esophageal cancer are shown in Figure 3. Table 2 presents the dosimetric comparison between the techniques according to the indices and parameters. In addition, the doses received by organ at risks in the region are presented in Table 3.

According to Table 2, the maximum dose and mean dose to the PTV were significantly reduced using the FIF technique ( $p<000$ and $p<0.003$, respectively). However, there was no significant difference in terms of the minimum dose. In terms of $\mathrm{V}>107 \%$, there was no difference between the techniques. However, D2 in the FIF technique was 
International Journal of Hematology and Oncology

\begin{tabular}{|ll|}
\hline $\begin{array}{l}\text { Table 1. Demographic characteristics of the patients and } \\
\text { data on PTV volumes. }\end{array}$ \\
\hline Characteristics (Mean $\left.\pm \mathrm{SD}^{\star}\right)$ & Esophagus $(\mathrm{n}=20)$ \\
Age (years) & $59.3 \pm 12.3$ \\
Weight (kg) & $61.2 \pm 11.7$ \\
Height (cm) & $168.7 \pm 9.3$ \\
BMl (kg.cm $\left.{ }^{-2}\right)$ & $21.6 \pm 5.1$ \\
PTV volume (cm3) & $312.8 \pm 80.7$ \\
\hline * Standard deviation (SD) & \\
\hline
\end{tabular}

significantly lower than 3D-CRT $(\mathrm{p}<0.001)$ and D98 in the FIF technique which was significantly higher than the 3D-CRT technique $(\mathrm{p}<0.004)$. V< 95\% in the FIF technique was less compared to the 3D-CRT technique $(\mathrm{p}<0.012)$ (Table 2).

The FIF technique allowed more homogeneous dose distributions compared to the 3D-CRT technique. The The HI mean values were $0.119 \pm 0.026$ and $0.0545 \pm 0.0128$ for the 3-D CRT and FIF tech-

\begin{tabular}{|c|c|c|c|}
\hline $\begin{array}{l}\text { Para- } \\
\text { meters }\end{array}$ & $\begin{array}{l}\text { 3D-CRT } \\
\left(\text { Mean } \pm S D^{\star}\right)\end{array}$ & $\begin{array}{l}\text { FIF } \\
\left(\text { Mean } \pm S D^{\star}\right)\end{array}$ & $p$ value \\
\hline $\mathrm{D}_{\text {Mean }}$ & $5275.50 \pm 19.41$ & $5239.73 \pm 20.29$ & 0.003 \\
\hline$D_{\operatorname{Max}}$ & $5816.67 \pm 106.91$ & $5528.98 \pm 138.79$ & 0.000 \\
\hline$D_{\text {Min }}$ & $4918.23 \pm 72.22$ & $4918.66 \pm 47.60$ & 0.984 \\
\hline$D_{2}$ & $110.8 \pm 2.94$ & $104.8 \pm 1.49$ & 0.001 \\
\hline$D_{98}$ & $98.79 \pm 0.90$ & $99.41 \pm 0.63$ & 0.004 \\
\hline$V>107 \%$ & $0.00 \pm 0.00$ & $0.00 \pm 0.00$ & - \\
\hline$V<95 \%$ & $1.85 \pm 0.75$ & $1.32 \pm 0.59$ & 0.012 \\
\hline $\mathrm{Cl}$ & $0.94 \pm 0.079$ & $0.97 \pm 0.46$ & 0.140 \\
\hline $\mathrm{HI}$ & $0.119 \pm 0.026$ & $0.0545 \pm 0.0128$ & 0.001 \\
\hline $\mathrm{MU}_{\text {Total }}$ & $241.7 \pm 18.31$ & $239.3 \pm 5.42$ & 0.174 \\
\hline
\end{tabular}

niques, respectively $(\mathrm{p}<0.001)$. However, there was no significant difference in CI between the two techniques.

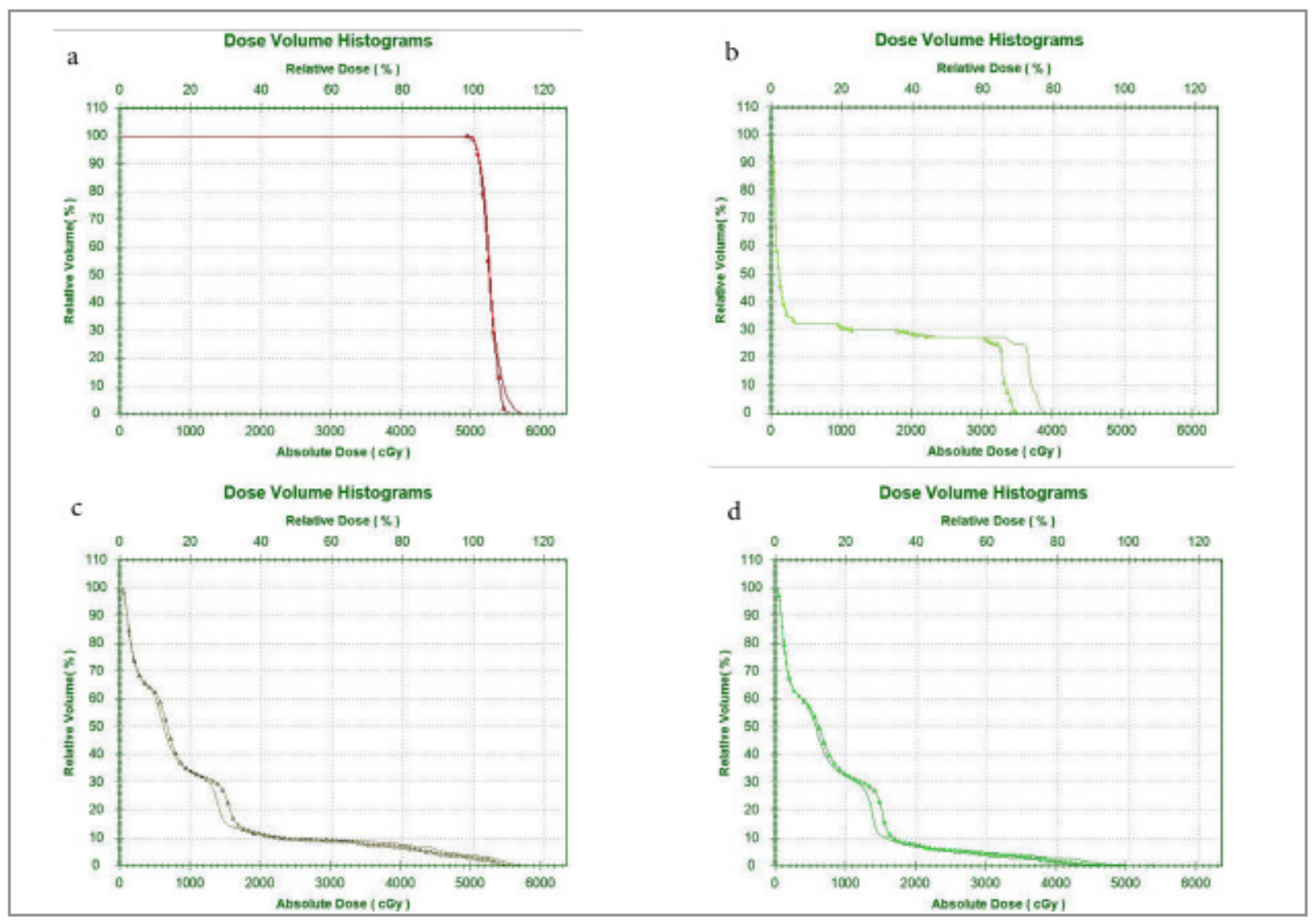

Figure 2. Comparisons of dose-volume histograms of wedge field technique versus FIF for esophageal cancer. (a) The PTV dosevolume histogram; (b) The spinal cord (OAR) dose-volume histogram; (c) The Right lung (OAR) dose-volume histogram; (d) The left lung (OAR) dose-volume histogram. Dotted line is related to Field-in-field technique. 


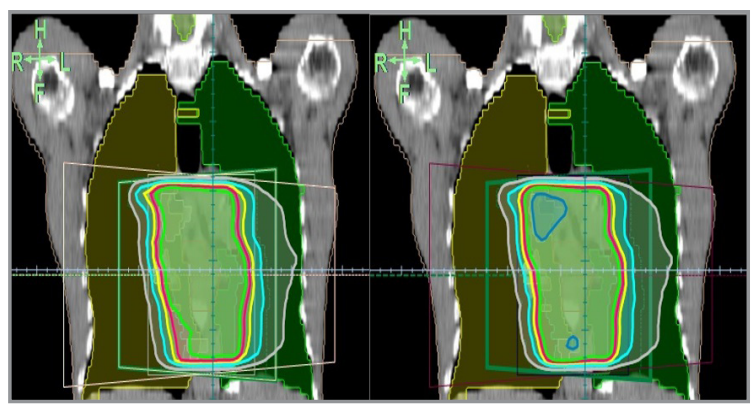

Figure 3. The isodose distributions of 3D-CRT and FIF treatment planning in esophageal cancer. Right side figure is 3DCRT and left side figure is field in field techniques isodose distribution.

The mean MU value \pm standard deviation required for the 3D-CRT and FIF techniques were $241.70 \pm 18.31$ and $239.30 \pm 5.42$, respectively. The difference in the average MU values used in the 3D-CRT and FIF techniques was not statistically significant $(\mathrm{p}=0.174)$ (Table 2$)$.

When the doses received by OARs were compared, they were in favor of the FIF technique. However, with regards to the heart, there was no significant difference between both techniques $(\mathrm{p}=0.154)$ (Table 3).

\section{DISCUSSION}

A number of studies have demonstrated the dosimetric benefits of FIF compared with the 3D-CRT technique for breast cancer studies and there are few studies on the feasibility of this technique in other parts of the body. ${ }^{11,21}$ This study demonstrated that, in general, the FIF technique had a better dosimetric outcome concerning the PTV mean dose and maximum dose, $\mathrm{HI}, \mathrm{D}_{2}, \mathrm{D}_{98}$, and $\mathrm{V}<95 \%$ than the 3D-CRT. However, there was no significant difference in the minimum dose, $\mathrm{V}>107 \%, \mathrm{CI}$ and MU between the two techniques.

The present study demonstrated the following as the advantages, when using the FIF technique: it reduced the scattered dose to patient, some hot spots that might persist in the 3D-CRT technique were avoided by adopting the FIF technique, maintaining the same gantry angles for each set of multiple fields ensured that there was no increase in setup complexity and that treatment can be delivered quickly and reliably., ${ }^{9,10,12}$

In recent years, there has been rapid improvement in the software and hardware of linear accelerators. ${ }^{21}$ The MLC is used instead of wedges for treatment techniques. When MLC was used, there was a decrease in the dose which scattered to unnecessary parts of the body. ${ }^{22,23}$

Our data showed that the FIF technique significantly reduced the maximum and mean doses of the treated region; however, in terms of minimum dose, there was no superiority in the application of the FIF technique. Prabhakar et al. ${ }^{10}$ compared these two techniques in different sites of the body and concluded that, for all the cases, the FIF technique was better than the 3D-CRT technique in terms of the maximum and mean doses. In their study, Yavas et al..$^{21}$ reported that the FIF technique, compared to the 3D-CRT for the whole breast radiotherapy caused a significant decrease in the maximum dose and mean dose of the PTV. In their study, Allaveisi et al. ${ }^{24}$ compared the 4-field conformal radiotherapy and FIF radiotherapy of the esophagus and found that FIF causes reduction in the maximum dose of PTV. Therefore, in terms of these parameters, the result of the present study is in agreement with those of previous studies. ${ }^{21,25,26}$

D2 was lower in the FIF technique and it caused an increase in the dose homogeneity of the target volume. In both techniques, V> 107\% was the same and these results confirmed the study done by Prabhakar et al. ${ }^{9,10}$

\begin{tabular}{|llll|}
\hline \multicolumn{3}{|l|}{ Table 3. Dosimetric comparison of doses of organ at risks (OARs) } & \\
\hline Organs at Risk & 3D-CRT (Mean \pm SD*) & FIF (Mean \pm SD) & p value \\
\hline Right Lung (V20) & $13.4 \pm 6.8$ & $8.8 \pm 3.1$ & 0.032 \\
Left Lung (V20) & $19.3 \pm 4.2$ & $12.2 \pm 5.1$ & 0.021 \\
Spinal Cord (Dmax) & $3541.02 \pm 487.65$ & $3260.90 \pm 546.19$ & 0.001 \\
Heart (V30) & $42.8 \pm 7.2$ & $39.2 \pm 6.8$ & 0.154 \\
\hline * Standard deviation (SD) & & & \\
\hline
\end{tabular}


In the present study, D98 was significantly higher for the FIF technique compared to the 3D-CRT technique. This result was in conflict with the results of Prabhakar et al.'s study. ${ }^{9}$

In the FIF technique, $\mathrm{V}<95 \%$ was less compared to 3D-CRT in the present study, which corroborates the findings by Hidekazu Tanaka et al. ${ }^{25}$ In their investigation, it was shown that the FIF technique had less $\mathrm{V}<95 \%$ for the FIF breast radiotherapy technique.

A comparison of $\mathrm{HI}$ between the plans showed statistically significant results with the FIF plans, confirming the findings of Baycan et al. ${ }^{13}$, Yavas et al. ${ }^{26}$, Ercan et al. ${ }^{12}$, and Allaveisi et al. ${ }^{24}$ who compared the two techniques and showed significant improvement in HI with the FIF technique. However in the field of CI, there was no significant difference between the two techniques, which was in agreement with the previous study by Baycan et al. ${ }^{13}$

In terms of total MU, the present study did not show any significant difference between the two techniques, which was in agreement with the previous study by Chui et al. ${ }^{27}$ In their study, Allaveisi et al. ${ }^{24}$ found a significant difference between both techniques, because in other studies wedge-based radiation fields are used in the 3DCRT technique, ${ }^{21,28}$ which can be explained by the fact that MU doses are higher with wedge-based plans because of radiation scatter. But in this study, radiation fields were used that did not have wedge; thus, the difference between this study and others could be understood. However, li-Min Sun et al. compared these two techniques for breast cancer radiotherapy and showed that the FIF technique had a greater total MU compared to the 3D-CRT technique and their results were in conflict with those of other studies in breast regions. ${ }^{28}$

The doses received by organs at risk in the present study were significantly reduced with the FIF technique. Prabhakar et al. ${ }^{9,10}$ showed that the FIF technique caused less maximum dose to organ at risks when compared with the 3D-CRT method. Also, other studies in other regions showed this reduction in dose to OARs, and it was in agreement with the present study.

Darby et al. ${ }^{29}$ reported that the risk of ischemic heart disease increases with increase in the dose received by the heart with a threshold of $7.4 \%$ per gray. Therefore, a reduction of the heart dose is very important in the radiation therapy of the thorax region.

\section{Conclusion}

The results of this study revealed that the use of the FIF technique depends on the complexity of the plan. It would be easier to use this technique when the number of beam fields is few; when the number of beams is more than three, it becomes time consuming for the planner. This study showed that the FIF technique had a superior dosimetric outcome to the 3D-CRT technique for esophageal cancer. There is no study on the application of the FIF technique in the esophageal region and most of the studies are on breast cancer. The FIF technique is a time consuming technique and it highly depends on the physicist's experience, knowledge and accuracy of the treatment planning system. Therefore, it can be expected that different results will be obtained in different studies. To the author's knowledge, the FIF technique is superior to 3D-CRT and it can also be used as a complementary technique to 3D-CRT, so as to improve the dosimetric results.

\section{Acknowledgements:}

The authors are grateful to the Vice Chancellor for Research (VCR) of Urmia University of Medical Sciences, for approving and supporting this project. The authors also express their gratitude to the physicians and staff of the Omid research and treatment center, for supporting the present study.

\section{REFERENCES}

1. Sadjadi A, Marjani H, Semnani S, Nasseri-Moghaddam S. Esophageal cancer in Iran: A review. Middle. East $J$ Cancer 1: 5-14, 2010.

2. Harirchi I, Kolahdoozan S, Hajizadeh S, et al., Esophageal cancer in Iran; a population-based study regarding adequacy of cancer surgery and overall survival. Eur J Surg Oncol 40: 352-357, 2014.

3. Zhang Y. Epidemiology of esophageal cancer. World J Gastroenterol 19: 5598-5606, 2013.

4. van Hagen P, Hulshof MC, Van Lanschot JJ, et al. Preoperative chemoradiotherapy for esophageal or junctional cancer. N Engl J Med 366: 2074-2084, 2012. 
5. Cooper JS, Guo MD, Herskovic A, et al. Chemoradiotherapy of locally advanced esophageal cancer: long-term follow-up of a prospective randomized trial (RTOG 85-01). JAMA 281: 1623-1627, 1999

6. Mahmoudi R, Jabbari N, Aghdasi M, Khalkhali HR. Energy dependence of measured CT numbers on substituted materials used for CT number calibration of radiotherapy treatment planning systems. PLoS ONE 11(7); e0158828, 2016.

7. Teniere P, Hay JM, Fingerhut A, Fagniez PL. Postoperative radiation therapy does not increase survival after curative resection for squamous cell carcinoma of the middle and lower esophagus as shown by a multicenter controlled trial. French University Association for Surgical Research. Surg Gynecol Obstet 173: 123-130, 1991.

8. Fok M, Sham JS, Choy D, et al. Postoperative radiotherapy for carcinoma of the esophagus: a prospective, randomized controlled study. Surgery 113: 138-147, 1993.

9. Prabhakar R, Julka PK, Rath GK. Can field-in-field technique replace wedge filter in radiotherapy treatment planning: a comparative analysis in various treatment sites. Australas Phys Eng Sci Med 31: 317-324, 2008.

10. Prabhakar R, Haresh KP, Kumar M, et al. Field-in-field technique for upper abdominal malignancies in clinical radiotherapy. J Cancer Res Ther 5: 20-23, 2009.

11. Sun LM, Meng FY, Yang TH, Tsao MJ. Field-in-field plan does not improve the dosimetric outcome compared with the wedged beams plan for breast cancer radiotherapy. Med Dosim 39: 79-82, 2014.

12. Ercan $\mathrm{T}$, Igdem $\mathrm{S}$, Alço $\mathrm{G}$, et al., Dosimetric comparison of field in field intensity-modulated radiotherapy technique with conformal radiotherapy techniques in breast cancer. Jpn J Radiol 28: 283-289, 2010.

13. Baycan D, Karacetin D, Balkanay AY, Barut Y. Field-in-field IMRT versus 3D-CRT of the breast. Cardiac vessels, ipsilateral lung, and contralateral breast absorbed doses in patients with left-sided lumpectomy: a dosimetric comparison. Jpn J Radiol 30: 819-823, 2012.

14. LinaTech, TiGRT TPS Radiation Treatment Planning System. Available from: www.linatech.com/product/tps.aspx. Accessed date: Aug 15, 2017.

15. Prescribing IC. Recording and reporting photon beam therapy (supplement to ICRU report 50). ICRU report, 1999.

16. Wambersie A, Landgerg T. ICRU report 62: prescribing, recording and reporting photon beam therapy. ICRU Publ Bethesda MD. 1999.

17. Halperin EC, Brady LW, Perez CA, Wazer DE. Perez \& Brady's principles and practice of radiation oncology. 6th Edition. Philadelphia, Lippincott Williams \& Wilkins; 2013: 203210.

18. Khan FM, Gerbi BJ. Treatment planning in radiation oncology. 4th edition. Philadelphia, Wolters Kluwer Health/Lippincott Williams \& Wilkins, 2016:987-994.

19. Gursel B, Meydan D, Ozbek N, Ofluoglu T. Dosimetric comparison of three different external beam whole breast irradiation techniques. Adv Ther 28: 1114-1125, 2011.
20. Kataria T, Sharma K, Subramani V, et al. Homogeneity Index: An objective tool for assessment of conformal radiation treatments. J Med Phys 37: 207-213, 2012.

21. Yavas G, Yavas C, Acar H, et al., Dosimetric comparison of 3-dimensional conformal and field-in-field radiotherapy techniques for the adjuvant treatment of early stage endometrial cancer. Phys Med 29: 577-582, 2013.

22. Stelzer KJ, Bailey B, Davidson M, et al. Determination of critical dosimetric parameters for breast radiation using forwardplanned segmented fields for intensity modulation. Med Dosim 32: 23-32, 2007.

23. Bhatnagar AK, Brandner E, Sonnik D, et al., Intensity modulated radiation therapy (IMRT) reduces the dose to the contralateral breast when compared to conventional tangential fields for primary breast irradiation. Breast Cancer Res Treat 96: 41-46, 2006.

24. Allaveisi F, Moghadam AN. Comparison between the fourfield box and field-in-field techniques for conformal radiotherapy of the esophagus using dose-volume histograms and normal tissue complication probabilities. Jpn J Radiol 1-8, 2017. 35: 327-334, 2017.

25. Tanaka H, Hayashi S, Kajiura Y, et al., Evaluation of the fieldin-field technique with lung blocks for breast tangential radiotherapy. Nagoya. J Med Sci 77: 339-345, 2015.

26. Yavas G, Yavas C, Acar H. Dosimetric comparison of whole breast radiotherapy using field in field and conformal radiotherapy techniques in early stage breast cancer. Int J Radiat Res 10: 131-138, 2012.

27. Chui CS, Hong L, Hunt M, McCormick B. A simplified intensity modulated radiation therapy technique for the breast. Med Phys 29: 522-529, 2002.

28. Sun LM, Meng FY, Yang TH, Tsao MJ. Field-in-field plan does not improve the dosimetric outcome compared with the wedged beams plan for breast cancer radiotherapy. Med Dosim 39: 79-82, 2014.

29. Darby SC, Ewertz M, McGale P, et al. Risk of ischemic heart disease in women after radiotherapy for breast cancer. $\mathrm{N}$ Engl J Med 368: 987-98, 2013.

\section{Correspondence:}

Nasrollah JABBARI

Solid Tumor Research Center

Department of Medical Physics and Imaging

Urmia University of Medical Sciences

URMIA / IRAN

Tel: 00984432752298

Fax: 00984432770047

e-mail: njabbarimp@gmail.com 